Bryn Mawr College

Scholarship, Research, and Creative Work at Bryn Mawr

College

Physics Faculty Research and Scholarship

Physics

1995

\title{
Solid state proton spin relaxation and methyl reorientation in isopropylbenzene
}

Amy L. Plofker

Peter A. Beckmann

Bryn Mawr College, pbeckman@brynmawr.edu

Let us know how access to this document benefits you.

Follow this and additional works at: https://repository.brynmawr.edu/physics_pubs

Part of the Physics Commons

\section{Custom Citation}

Plofker, Amy L. and Peter A. Beckmann. 1995. "Solid state proton spin relaxation and methyl reorientation in isopropylbenzene." Journal of Physical Chemistry 99.1: 391-394.

This paper is posted at Scholarship, Research, and Creative Work at Bryn Mawr College. https://repository.brynmawr.edu/physics_pubs/127

For more information, please contact repository@brynmawr.edu. 


\title{
Solid-State Proton Spin Relaxation and Methyl Reorientation in Isopropylbenzene
}

\author{
Amy L. Plofker and Peter A. Beckmann* \\ Department of Physics, Bryn Mawr College, 101 North Merion Avenue, Bryn Mawr, Pennsylvania 19010-2899 \\ Received: July 1, $1994^{\otimes}$
}

We have measured the temperature and Larmor frequency dependence of the proton spin-lattice and spinspin relaxation rates in solid isopropylbenzene. The sample melts at too low a temperature to observe the high-temperature frequency-independent regime from which a rotational barrier is normally extracted. By measuring the rates at three Larmor frequencies, however, we demonstrate that all the relevant parameters that characterize methyl reorientation can be overdetermined within the confines of a given dynamical model even though the model may not be unique. The relaxation technique is very sensitive to the state of the solid, and interesting and unusual thermal history effects are presented and discussed.

\section{Introduction}

Solid-state proton spin relaxation studies have provided considerable insight into the general problem of internal motions in molecules. In this paper, we investigate methyl group reorientation and molecular structure in solid isopropylbenzene (Figure 1). Following a brief theory review appropriate to motion in alkyl-substituted organic molecules like isopropylbenzene, we present the Experiments section. This section is divided into three parts. The first part succinctly presents the data, whereas the second and third parts discuss thermal history effects in considerable detail. The Data Analysis and the Discussion and Summary sections then return to the interpretation of the data in terms of the intramolecular motion and molecular structure.

\section{Theory Review}

The spin-lattice relaxation rate $R_{1}$ is given by an appropriate sum $R_{1}=\sum_{i} A_{i}\left[J\left(\omega, \tau_{i}\right)+4 J\left(2 \omega, \tau_{i}\right)\right]$ for strength factors $A_{i}$ and spectral density $J .{ }^{1-5}$ The Larmor angular frequency $\omega=\gamma B$ for magnetic field $B$ and proton magnetogyric ratio $\gamma$ characterizes the resonant exchange between the spin system and its environment. The correlation times $\tau_{i}$ characterize the dynamics of the reorientation process. The spectral density often takes on the unique- $\tau$ form: $J_{\mathrm{BP}}\left(\omega, \tau_{\mathrm{BP}}\right)=2 \tau_{\mathrm{BP}}\left(1+\omega^{2} \tau_{\mathrm{BP}^{2}}\right)^{-1}$ with $\tau_{\mathrm{BP}}=\tau_{\infty} \exp (V / k T)$ for reorientation barrier $V$ and temperature $T$. The subscript BP on $\tau$ refers to Bloembergen, Purcell, and Pound, who first used this form in their classic paper. ${ }^{6}$ This form for $J$ follows naturally from the assumption of random motion (Poisson statistics) and a unique barrier $V$. If there is a distribution of barriers, a distribution of $\tau$ values results, and the form of $J$ becomes model dependent. (In addition, it is possible that there is a unique barrier but that the mution is not random. In this case, the nonexponential correlation function can be expressed as a distribution of exponential correlation functions, and a distribution of correlation times results. Although the underlying model is physically very different, the mathematical formalism is the same as for a real, physical distribution of correlation times.) The unique- $\tau$ form for the spectral density contains the essence of much of the basic physics ${ }^{1,2}$ and can serve as the purpose of general discussion. Details have been presented for the superposition of methyl and ethyl group reorientation ${ }^{4}$ and for the superposition of methyl and tert-butyl group reorientation. ${ }^{5}$ The changes from these

\footnotetext{
${ }^{\otimes}$ Abstract published in Advance ACS Abstracts, November 15, 1994.
}

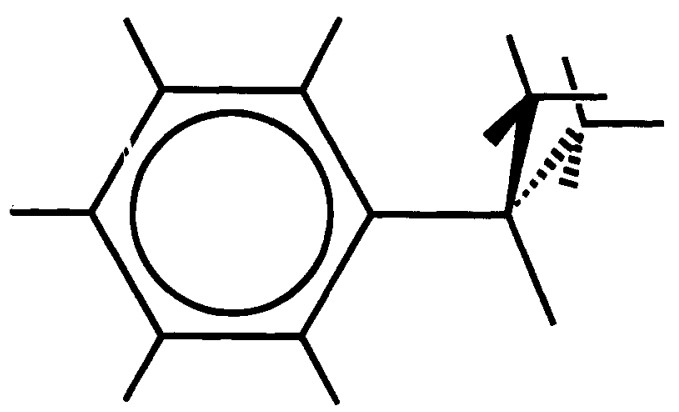

Figure 1. Schematic picture of isopropylbenzene.

presentations for the superposition of methyl and isopropyl group reorientation are straightforward. ${ }^{7} \ln \left(R_{1}\right)$ vs $T^{-1}$ has a characteristic maximum when $\omega \tau$ is near unity. When a hightemperature solid state is available, $R_{1}$ is independent of $\omega$ at high temperatures $(\omega \tau \ll 1)$, as can be seen in ethyl ${ }^{4}$ and other isopropy $1^{8}$ substituted benzenes. This is true even if complicated spectral densities, characteristic of a distribution of correlation times, ${ }^{3}$ are used. ${ }^{4,8}$ At low temperatures, $R_{1}(\omega, T)$ is often very sensitive to the form of the spectral density. Indeed, it is a quite general consequence of most stochastic processes that the $\omega \tau \gg 1$ regime is more sensitive to the probability distribution functions than is the $\omega \tau \ll 1$ regime. $^{9}$ If both the low- and high-temperature regimes can be mapped out at several Larmor frequencies $\omega /(2 \pi)$, a great deal can be learned about the spectral density and, subsequently, about the reorientation of the alkyl group (tert-butyl, ${ }^{5,10-12}$ isopropyl,$^{8}$ or ethyl $1^{4}$ ) and its constituent methyl group or groups. The high-temperature frequencyindependent $R_{1}$ data act like a standard Arrhenius plot, and the slope of $\ln \left(R_{1}\right)$ vs $T^{-1}$ gives the barrier $V$ independent of the form of the spectral density (for all spectral densities that have been found to be applicable). ${ }^{3}$ For a spectral density that arises either from a nonrandom process or from a distribution of $\tau$ values (each characterizing a set of random rotors), the barrier extracted from the data is some well-defined barrier characteristic of the dynamical process. ${ }^{3}$

\section{Experiments}

1. Spin-Lattice and Spin-Spin Relaxation Rate Measurements. The spin-lattice relaxation rate $R_{1}$ and the spinspin relaxation rate $R_{2}$ were measured between about 100 and $150 \mathrm{~K}$. $R_{1}$ was measured at $\omega /(2 \pi)=8.50,22.5$, and $53.0 \mathrm{MHz}$ using a standard $\pi-t-\pi / 2-t^{\prime}$ pulse sequence with $t^{\prime} \geq 10 R_{1}^{-1}$ to ensure equilibrium before each new measurement. The 


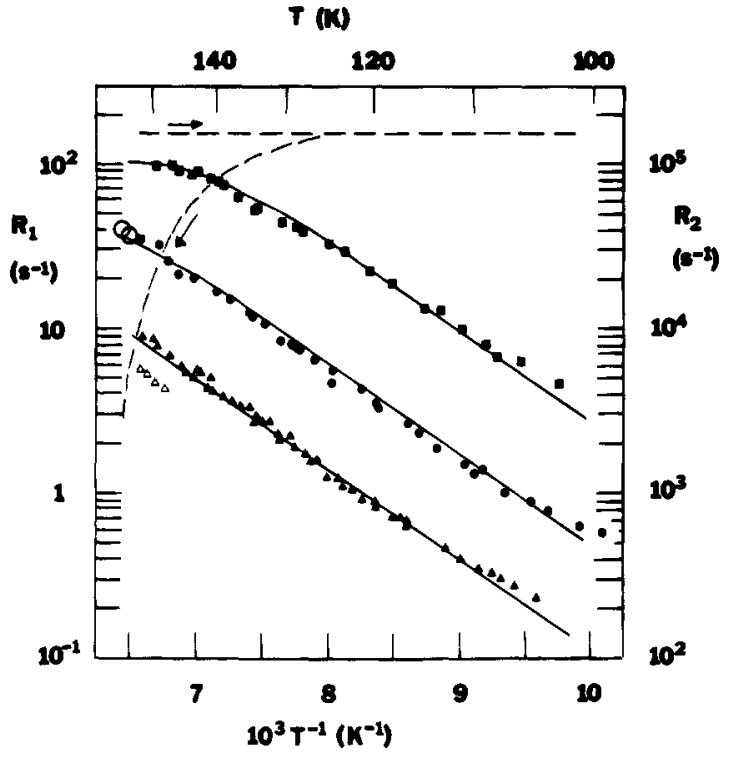

Figure 2. Temperature $T$ dependence of the spin-lattice relaxation rate $R_{1}$ (left axis) and the spin-spin relaxation rate $R_{2}$ (right axis) in isopropylbenzene. Experimental data are shown for $R_{1}$ at Larmor frequencies of $8.50(\square), 22.5(\bullet, O)$, and $53.0 \mathrm{MHz}(\Delta, \Delta)$. The solid symbols $(\mathbf{\square}, \mathbf{Q}, \mathbf{\Delta})$ indicate the usual solid state as discussed in the text. The open triangles $(\Delta)$ indicate an unusual solid state as discussed in the text. The open circles $(O)$ indicate the liquid state. The three solid lines show a single fit to the $R_{1}$ data as discussed in the text. Experimental data for $R_{2}$ at frequencies of 22.5 and $53.0 \mathrm{MHz}$ are represented by the dashed lines. $R_{2}$ is independent of frequency. The arrows indicate whether $R_{2}$ was measured on increasing or decreasing the temperature.

details are discussed elsewhere. ${ }^{10}$ The spin-lattice relaxation process was exponential; thus, $R_{1}$ is uniquely defined. The values are shown in Figure 2. The experimentally determined uncertainty in each $R_{1}$ value is about $\pm 5 \%$, which is smaller than the spread in adjacent data points in Figure 2, indicating the presence of either systematic errors or residual effects of thermal history as discussed below.

The free induction decay (fid) was typical of solids, ${ }^{1}$ and the spin-spin relaxation rate $R_{2}$ was measured, at 8.50 and 53.0 $\mathrm{MHz}$, from the middle region of the nonexponential fid. This corresponds to about $3-4 \mu \mathrm{s}$ after the $\pi / 2$ pulse for $R_{2}$ values of $1.5 \times 10^{5} \mathrm{~s}^{-1}\left(T_{2}=7 \mu \mathrm{s}\right) . R_{2}$ was independent of frequency and is indicated by the dashed line in Figure 2 for the cases of decreasing and increasing temperature. The absolute values of these representative $R_{2}$ vs $T$ lines are not so meaningful given the nature of the free induction decay and the arbitrary determination of $R_{2}$. However, the thermal history of $R_{2}$ so defined could be accurately reproduced.

2. Sample Preparation and Thermal History Effects. The proton spin relaxation technique is very sensitive to the state of the solid and to thermal history effects. The data in Figure 2 result from using several carefully prepared samples. The isopropylbenzene was obtained from Aldrich Chemical Co., and the quoted purity was $99 \%$. The quoted freezing point was 177 $\mathrm{K}$, consistent with published values. The samples were liquid at room temperature. They were degassed using different numbers of freeze-pump-thaw cycles and then sealed. $R_{1}$ vs $T$ was the same for all samples when thermal history was kept the same. When slowly frozen, the freezing point was $154 \mathrm{~K}$.

The several samples were frozen in a variety of ways and treated to a variety of thermal histories to check the thermal history dependence of $R_{1}$ and $R_{2}$ vs $T$. (The room-temperature liquid samples were taken both rapidly and slowly to the solid state at $150 \mathrm{~K}$, or to $77 \mathrm{~K}$, or in between and then, sometimes, taken up and down in temperature in the solid state both rapidly and slowly, etc., before $R_{1}$ and $R_{2}$ were measured.) The $R_{1}$ data indicated by solid squares, circles, and triangles in Figure 2 represent many thermal histories with several samples used over a period of years. Within experimental uncertainty, these $R_{1}$ values (solid symbols) have a very slight dependence, if any at all, on thermal history.

On 2 occasions (out of 30 or so), we obtained the $53-\mathrm{MHz}$ $R_{1}$ vs $T$ data shown by the 4 high-temperature open triangles. These data were obtained by starting from the solid (in the NMR probe) and slowly heating until the sample was liquid. On some occasions, the existence of the liquid state was determined by noting that $100 \%$ of the signal was typical of a liquid with an $R_{2}$ characteristic of the magnet inhomogeneity. On other occasions, this was determined by visual inspection (which ended the experiment). The crucial step is that the temperature of the liquid was never taken to a value higher than a few degrees above the point where the whole sample had melted. The cold liquid sample was then slowly cooled to the $100 \%$ solid state (no liquid component to the NMR line), and the open triangles in Figure 2 were obtained. If a sample was frozen from room temperature (slowly or quickly) or if the (recently previously solid) liquid was taken to too high a temperature, data characteristic of the closed symbols were found. This seems to imply that the cold liquid remembered its previous solid state so long as the temperature was not taken to too high a value. Indeed, it was perhaps a glass with a viscosity and an NMR line width (and a visual appearance!) characteristic of an isotropic liquid. This is unusual. These kinds of experiments are certainly not the best way to study these phenomenon, and others, better equipped to investigate these effects, are encouraged to do so. We feel that it is important to mention this unusual effect even though we are unable to perform a quantitative analysis or obtain a quantitative result concerning the structure from this aspect of the experiment.

Early exploratory experiments on undegassed, unsealed samples resulted in $R_{1}$ vs $T$ data that were literally "all over the place". These data are not shown in Figure 2. One always found a nice smooth curve on any given day's run (i.e., a particular thermal history), but the next day's $R_{1}$ vs $T$ curve could differ by as much as a factor of 3 in $R_{1}$. A preliminary report of an extreme case of these kinds of effects has been presented. ${ }^{13}$ It is important to note here that if one did a single, very lengthy experiment involving a single down and up sweep in temperature with a poorly prepared sample, one would obtain a very smooth set of data with relatively little scatter. It would, however, be only one of several possible very different sets of data. We suspect that such reports are commonplace.

3. Thermal Histories of $R_{1}$ and $R_{2}$, Intermolecular Barriers, and Methyl Reorientation. Two effects are at work in producing these thermal history effects. Crudely, $R_{1}=A J$ (as presented in the Introduction). The motion determines the spectral density $J$, and the rms strengths of the spin-spin interactions determine the strength parameter $A$. First, we assume a nonrandom motion spectral density $J$ results from a distribution of correlation times. $J$ is sensitive to this distribution, which depends on the state of the solid; the more crystalline, the narrower the distribution. In turn, the correlation time depends on the height of the barrier for methyl reorientation and on its shape. Although the unique intraalkyl (electronic) barrier certainly dominates the total barrier, as discussed below, intermolecular electrostatic interactions will contribute to the total barrier and its shape. Indeed, the intermolecular component may well contribute in either a positive or negative sense, depending on how pairs of molecules fit together, and this can 
change dramatically between the crystalline and amorphous states. That is, depending on the geometry of the solid state, intermolecular interactions can raise the maxima of the potential function (thus increasing the barrier) or raise the minima of the potential function (thus reducing the barrier). An interesting example of the possibility of the latter is 1,4-diethylbenzene. ${ }^{4}$

Second, intermolecular proton spin-proton spin interactions sometimes play a significant role in determining the value of $A$ in $R_{1}=A J$. These interactions usually play a very significant role in determining the value of $R_{2}{ }^{1,2}$ The latter is doubtless true for isopropylbenzene in all its solid phases. However, if methyl group reorientation is the only motion on the $\omega^{-1}$ time scale, only protons near the methyl protons have an effect in increasing $A$ from a value obtained from considering only intramethyl spin-spin interactions. For the case of methyl groups, the intramethyl interactions usually dominate due to the $r^{-6}$ dependence (for proton-proton separation $r$ ) of the dipolar

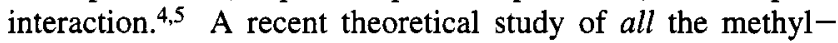
non-methyl proton spin-spin interactions in methyl-substituted planar aromatic molecules bears this out. ${ }^{14}$ Indeed, we are able to estimate that all intramolecular interactions involving a methyl proton and a proton not in the same methyl group will increase $A$ (and thus $R_{1}$ ) in isopropylbenzene by $5-15 \%$ over the value obtained considering intramethyl interactions only.

It is unlikely that intermolecular proton-proton interactions play a significant role for isopropylbenzene, given the characteristic structures of these kinds of organic solids. ${ }^{15}$ We discuss this effect in some detail because the proton spin relaxation technique is often severely criticized because of the unknown magnitude of intermolecular spin-spin interactions. Whereas these interactions can be very significant in some cases (like solid benzene ${ }^{16-18}$ ), they are rarely dominant in methylsubstituted systems.

The fact is that $R_{1}$ can depend on the structure of the solid in a very sensitive manner via the dependence of the spectral density on the correlation time. Many careful experiments must be performed on carefully prepared samples in order to zero in on the "desired" or the "average" or at least a "typical" structure. Impurities may control the crystallization process and the resulting state of the polycrystalline or amorphous solid. The two highest temperature data points at $22.5 \mathrm{MHz}$, indicated by $O$ 's in Figure 2, are in the liquid state. That these values are on the same curve as the solid-state data strongly suggests that the motions on the $\omega^{-1}$ time scale in the solid and the cold liquid phases are the same. This is most unusual. Normally, at a phase transition, one sees significant discontinuities, either in the $R_{1}$ values themselves or in the slope of $\ln \left(R_{1}\right)$ vs $T^{-1}$.

On slowly decreasing the temperature from the liquid state, $R_{2}$ was about $1.5 \times 10^{5} \mathrm{~s}^{-1}$ in the solid below $154 \mathrm{~K}$, as indicated by the horizontal dashed line in Figure 2. This value remained constant to the lowest temperatures and was independent of Larmor frequency. This "rigid-lattice" value of $R_{2}{ }^{-1}$ $=T_{2}=7 \mu$ s is typical of organic solids with many relatively closely-spaced protons. On increasing temperature, $R_{2}$ began to decrease at about $130 \mathrm{~K}$, as indicated in Figure 2. It decreased rapidly to the very small value determined by the inhomogeneities of the magnets as the liquid transition near $154 \mathrm{~K}$ was approached. Unlike the $R_{2}$ values, the $R_{1}$ values indicated by the solid symbols in Figure 2 did not depend on whether temperature was increasing or decreasing. Although the open triangles in the vicinity of $150 \mathrm{~K}$ correspond only to the decreasing temperature experiments (where $R_{2}$ maintains the constant value of $1.5 \times 10^{5} \mathrm{~s}^{-1}$ ) and even then only if the sample had been previously solidified and not taken to too high a temperature as discussed above, the solid symbols for $R_{1}$ in that region correspond to both the decreasing (where $R_{2}=1.5 \times$ $10^{5} \mathrm{~s}^{-1}$ ) and increasing (where $R_{2}$ is about $2 \times 10^{4} \mathrm{~s}^{-1}$ ) temperature experiments. For reasons presented below, we will conclude from the $R_{1}$ values that only the methyl groups are moving on the $\omega^{-1}$ time scale. The $R_{2}$ values clearly show, however, that there are additional slower motions on increasing temperature.

\section{Data Analysis}

Even though the high-temperature $R_{1}$ vs $T$ region is not observable for isopropylbenzene (because the sample melts), the data in Figure 2 can be fitted because more than one Larmor frequency is available. Several models might work as discussed below, but here we use a Davidson-Cole spectral density 3,4 $\left.J_{\mathrm{DC}}\left(\omega, \tau_{\mathrm{DC}}, \epsilon\right)=2 \omega^{-1}\left[\sin \left\{\epsilon \arctan \left(\omega \tau_{\mathrm{DC}}\right)\right\}\right]\left[1+\omega^{2} \tau_{\mathrm{DC}}{ }^{2}\right]^{-\epsilon / 2}\right]$ because it has the fewest adjustable parameters that can fit these types of data (one more than the unique- $\tau$ spectral density), it is physically reasonable, and it allows us to compare the parameters with previous studies. The Davidson-Cole spectral density assumes a distribution of $\tau$ values given by $\Lambda_{\mathrm{DC}}\left(\tau ; \tau_{\mathrm{DC}}, \epsilon\right)$ $=[\sin (\epsilon \pi)] \pi^{-1} \tau^{-1+\epsilon}\left(\tau_{\mathrm{DC}}-\tau\right)^{-\epsilon}$ for $\tau \leq \tau_{\mathrm{DC}}$ and $\Lambda_{\mathrm{DC}}\left(\tau ; \tau_{\mathrm{DC}}, \epsilon\right)$ $=0$ for $\tau>\tau_{\mathrm{DC}}$. $J_{\mathrm{DC}}$ then follows from $J_{\mathrm{DC}}=\int_{0}^{\infty} \Lambda_{\mathrm{DC}}(\tau) J_{\mathrm{BP}}$ $(\tau) \mathrm{d} \tau$. The parameter $\tau_{\mathrm{DC}}$ is a cutoff correlation time. If $\Lambda_{\mathrm{DC}}$ is replaced by $\Lambda_{\mathrm{BP}}=\delta\left(\tau-\tau_{\mathrm{BP}}\right)$, where $\delta$ is a Dirac $\delta$ function, $J_{\mathrm{BP}}\left(\omega, \tau_{\mathrm{BP}}\right)$, presented in the Introduction, is recovered. The parameter $\epsilon$ characterizes the width of the distribution of $\tau$ values. As $\epsilon \rightarrow 1, \Lambda_{\mathrm{DC}} \rightarrow \Lambda_{\mathrm{BP}}$ and $J_{\mathrm{DC}} \rightarrow J_{\mathrm{BP}}$. Finally, it is assumed that $\tau_{\mathrm{DC}}$ is given by $\tau_{\mathrm{DC}}=\tau_{\infty} \exp (V / k T)$ and the four adjustable parameters are $V, \tau_{\infty}, \epsilon$, and $A$ with $R_{1}=A\left[J_{\mathrm{DC}^{-}}\right.$ $\left.\left(\omega, \tau_{\mathrm{DC}}, \epsilon\right)+4 J_{\mathrm{DC}}\left(2 \omega, \tau_{\mathrm{DC}}, \epsilon\right)\right]$. The $R_{1}(\omega, T)$ data in Figure 2 overdetermine these four parameters even though the hightemperature $\omega \tau \ll 1$ regime is not observed. One can think of the data at two frequencies as being fitted and the third being predicted. The fitted parameters are the barrier $V=14.2 \mathrm{~kJ}$ $\mathrm{mol}^{-1}, \tau_{\infty}=1.9 \times 10^{-13} \mathrm{~s}, \epsilon=0.74$, and $A=2.2 \times 10^{9} \mathrm{~s}^{-2}$. The uncertainties in $V, \epsilon, \tau_{\infty}$, and $A$ are about $\pm 5 \%, \pm 10 \%$, $\pm 25 \%$, and $\pm 10 \%$. A distribution of $V$ values for $\epsilon=0.85$ is presented elsewhere. ${ }^{4}$ The value $\epsilon=0.74$ determined here corresponds to a very narrow distribution of barriers at, and just below, $14 \mathrm{~kJ} \mathrm{~mol}^{-1}$.

The parameter $A$ can be compared to a theoretical value $\tilde{A}$ obtained from assuming (1) that only intramethyl dipole-dipole interactions are taken into account and (2) that the only motion on the $\omega^{-1}$ time scale is methyl group reorientation. In this case, $A / \tilde{A}=1.16 .^{3,4,7}$ In fact, this ratio would be closer to unity if other intramolecular spin-spin interactions were considered since they will contribute between $5 \%$ and $15 \%$ of the $\tilde{A}$ value determined from intramethyl interactions alone. ${ }^{14}$ Within the confines of the Davidson-Cole spectral density, this shows that only the methyl groups are reorienting on the $\omega^{-1}$ time scale. If we use $\tilde{A}$ for the superposition of isopropyl and methyl group reorientation, the ratio $A / \tilde{A}$ would be about 0.5 , and this completely rules out that model. ${ }^{7}$ We can conclude that in order to appear motionless on the $\omega^{-1}$ time scale in these relaxation experiments, the barrier for isopropyl group reorientation is greater than about $50 \mathrm{~kJ} \mathrm{~mol}^{-1}$. This value can be compared with a value of $1.0 \pm 0.6 \mathrm{~kJ} \mathrm{~mol}^{-1}$ obtained from gas-phase low-resolution microwave spectroscopy experiments in 3,5dibromoisopropylbenzene. 19 It can also be compared with the values of $8.2 \pm 0.8 \mathrm{~kJ} \mathrm{~mol}^{-1}$ in isopropylbenzene $\mathrm{e}^{20}$ itself and $21 \pm 7 \mathrm{~kJ} \mathrm{~mol}^{-1}$ in 2,6-difluoroisopropylbenzene, ${ }^{21}$ both obtained from liquid-state nuclear magnetic resonance studies of $J$ splittings. The difference here is that in the solid state the isopropyl groups are immobile on the $\omega^{-1}$ time scale and the solid-state proton spin-lattice relaxation technique can be used to study methyl group reorientation. 
Finally, the $\tau_{\infty}$ value can be compared with a theoretical value $\tilde{\tau}_{\infty}$ based on very simple but surprisingly successful models for methyl group reorientation. ${ }^{22}$ The ratio $\tau_{\infty} / \tilde{t}_{\infty}$ is $1.3 \pm 0.3$ in this case, and for example, if whole-molecule rotation were responsible for the relaxation, this ratio would be very different since the moments of inertia play an important role in this simple model.

$R_{1}$ data such as that presented in Figure 2 can easily be misinterpreted. For example, if the data are fitted independently with three straight lines and treated as Arrhenius plots, the slopes are $10 \mathrm{~kJ} \mathrm{~mol}^{-1}$ for the $22.5-$ and $53.0-\mathrm{MHz}$ data and $8.3 \mathrm{~kJ}$ $\mathrm{mol}^{-1}$ for the $8.50-\mathrm{MHz}$ data. In the more careful analysis presented here, the parameter $V$ is found to be $14.2 \pm 0.7 \mathrm{~kJ}$ $\mathrm{mol}^{-1}$. The first lesson is that the low-temperature $\ln \left(R_{1}\right)$ vs $T^{-1}$ data do not generally give the barrier. Indeed, for the Davidson-Cole distribution, the magnitude of the lowtemperature slope of $\ln \left(R_{1}\right)$ vs $T^{-1}$ is $\epsilon V$, which, for the fit presented above, is $10.5 \mathrm{~kJ} \mathrm{~mol}^{-1}$. The even lower value of $8.3 \mathrm{~kJ} \mathrm{~mol}^{-1}$ for the $8.50-\mathrm{MHz}$ data comes about because the maximum in $R_{1}$ (i.e., where $\omega \tau$ approaches unity) is being approached at the highest temperatures. If the high-temperature region is not observed, it is important to do experiments at low enough a Larmor frequency so that some curvature is observed. An additional prediction of the Davidson-Cole spectral density born out by the current experiments is that for $\omega \tau_{\mathrm{CD}} \gg 1, R_{1}$ is proportional to $\omega^{-(1-\epsilon)}$.

All the factors and requirements discussed above greatly restrict the family of spectral densities that will fit the data. ${ }^{3}$ Unfortunately, it is still unlikely that the fit to the DavidsonCole spectral density is unique. Other spectral densities will likely fit the data. However, if one assumes that the relaxation at high temperature $(\omega \tau \ll 1)$ would be independent of $\omega$ (where such data available), then only a very small family of spectral densities $^{3}$ would fit the data, and all would predict lowtemperature slopes analogous to $\epsilon V$ where $\epsilon$ was the ratio of slopes and $V$ was a barrier height. In many studies, we have never observed a $\omega \tau \ll 1 R_{1}$ vs $T$ region that depends on $\omega$ for methyl group reorientation.

\section{Discussion and Summary}

Fitting the $R_{1}$ vs $T$ data in isopropylbenzene with a Davidson-Cole spectral density gives a very narrow distribution of barriers for methyl group reorientation. These barriers are at and slightly lower than the cutoff barrier of $V=14 \mathrm{~kJ} \mathrm{~mol}^{-1}$. If a different dynamical model (i.e., a different spectral density) were used, the fitted barriers would not be very different. This value of $V$ can be compared with the value of $14 \mathrm{~kJ} \mathrm{~mol}^{-1}$ in 1,4-diisopropylbenzene, ${ }^{23} 13 \mathrm{~kJ} \mathrm{~mol}^{-1}$ in 1,3,5-triisopropylbenzene, ${ }^{23} 13 \mathrm{~kJ} \mathrm{~mol}^{-1}$ in ethylbenzene, ${ }^{4} 15 \mathrm{~kJ} \mathrm{~mol}^{-1}$ in $1,3-$ diethylbenzene, ${ }^{4}$ and $12 \mathrm{~kJ} \mathrm{~mol}^{-1}$ in 1,2-diethylbenzene. ${ }^{4}$ (With uncertainties of about $\pm 10 \%$, these barriers are all about the same.) In these ethyl- and isopropyl-substituted benzenes, the barrier for methyl reorientation is dominated by the intraalkyl electronic barrier of about $12 \mathrm{~kJ} \mathrm{~mol}^{-1}$ corresponding to about $4 \mathrm{~kJ} \mathrm{~mol}^{-1}$ per bond overlap (as in ethane ${ }^{24}$ ). The molecule 1,4-diethylbenzene ${ }^{4}$ is an interesting exception in that the "negative" contribution of the intermolecular barrier may be responsible for an observed barrier of only $9.3 \mathrm{~kJ} \mathrm{~mol}^{-1}$. In none of these cases does the alkyl group reorient on the $\omega^{-1}$ time scale in the solid state. The barriers presented here show that the methyl groups are well away from the aromatic plane. They also show that the other intramolecular interactions and the intermolecular interactions in the solid state usually (but not always) contribute no more than plus or minus a few $\mathrm{kJ}$ $\mathrm{mol}^{-1}$. This range of barriers is also found for many out-of- plane methyl groups in many tert-butylbenzenes. ${ }^{11,12}$ The most likely orientation of the isopropyl group in isopropylbenzene is the planar arrangement (Seeman et al. ${ }^{25}$ ) with the two methyl groups on opposite sides of the aromatic plane and the lone proton in the plane adjacent to a ring proton. This is the structure shown in Figure 1. Other geometries would result in a much greater contribution to $V$ from the intramolecular barrier. We are unable to comment on the equilibrium orientation of the methyl groups, although the basis on which to establish this has been presented. ${ }^{14}$

In summary, solid isopropylbenzene is most unusual from the proton spin relaxation experiment point of view. The technique is extremely sensitive to which motions are occurring and on the state of the solid. We have determined that in solid isopropylbenzene only the methyl groups are reorienting on the inverse Larmor frequency time scale and we are able to measure the barrier of about $14 \mathrm{~kJ} \mathrm{~mol}^{-1}$. Obtaining barriers in this range using other experimental techniques is very difficult. Obtaining these relatively small barriers by numerical techniques is also very difficult. Finally, solid isopropylbenzene seems to have some interesting and unusual solid states and thermal history effects. An investigation of these matters is best left to those better equipped to do materials science. Within the confines of a particular dynamical model, details of the crystalline or glassy structure do not affect the determination of the barrier for methyl group reorientation which is dominated by the intramolecular electronic barrier experienced by a methyl group in an isopropyl group.

\section{References and Notes}

(1) Abragam, A. The Principles of Nuclear Magnetism: Oxford University: Oxford, 1961.

(2) Slichter, C. P. Principles of Nuclear Magnetism; Springer: Berlin, 1978.

(3) Beckmann, P. A. Phys. Rep. 1988, 171, 85.

(4) Beckmann, P. A.; Happersett, L.; Herzog, A. V.; Tong, W. M. J. Chem. Phys. 1991, 95, 828.

(5) Beckmann, P. A.; Hathorn, R. M.; Mallory, F. B. Molec. Phys. $1990,69,411$.

(6) Bloembergen, N.; Purcell, E. M.; Pound, R. V. Phys. Rev. 1948 73,679 .

(7) Beckmann, P. A. Unpublished calculations.

(8) Al-Hallaq, H. A.; Beckmann, P. A. J. Chem. Soc., Faraday Trans. 1993, 89, 3801

(9) Reichl, L. E. A Modern Course in Statistical Mechanics; University of Texas: Austin, 1980.

(10) Beckmann, P. A.; Hill, A. I.; Kohler, E. B.; Yu, H. Phys, Rev. B 1988, 38, 11098 .

(11) Fry, A. M.; Beckmann, P. A.; Fry, A. J.; Fox, P. C.; Isenstadt, A. J. Chem. Phys. 1991, 95, 4778.

(12) Beckmann, P. A.; Cheung, A. M.; Fisch, E. E.; Fusco, F. A.; Herzog, R. E.; Narasimhan, M. J. Chem. Phys. 1986, 84, 1959.

(13) Beckmann, P. A. Physics and Chemistry of Finite Systems: From Clusters to Crystals; Jena, P., Khanna, S. N., Rao, B. K., Eds.; Kluwer Academic: Amsterdam, 1992; Vol. 1, p 357. 267.

(14) Palmer, C.; Albano, A. M.; Beckmann, P. A. Physica B 1993, 190 ,

(15) Kitaigorodsky, A. I. Molecular crystals and molecules; Academic: New York: 1973.

(16) van Steenwinkel, R. Z. Naturforsch. 1969, 24a, 1526.

(17) Andrew, E. R.; Eades, R. G. Proc. R. Soc. London 1953, $218,537$.

(18) Andrew, E. R. Physica 1951, 17, 405.

(19) True, N. S.; Farag, M. S.; Bohn, R. K.; MacGregor, M. A.; Radhakrishnan, J. J. J. Phys. Chem. 1983, 87, 4622.

(20) Parr, W. J. E.; Schaefer, T. Acc. Chem. Res. 1980, 13, 400

(21) Schaefer, T.; Veregin, R. P.; Laatikainen, R.; Sebastian, R.; Marat, K.; Charlton, J. L. Can. J. Chem. 1982, 60, 2611.

(22) Conn, K. G.; Beckmann, P. A.; Mallory, C. W.; Mallory, F. B. J. Chem. Phys. 1987, 87, 20.

(23) Beckmann, P. A.; Cheung, A. M.; Herzog, R. E.; Fisch, E. E.; Narasimhan, M.; Mallory, C. W.; Mallory, F. B. J. Chem. Soc., Faraday Trans. 2, 1985, 81, 1013 .

(24) Pine, S. H. Organic Chemistry; McGraw-Hill: New York: 1987.

(25) Seeman, J. I.; Secor, H. V.; Breen, P. J.; Grassian, V. H.; Bernstein. E. R. J. Am. Chem. Soc. 1989, 111, 3140 . 